\title{
TEM and STEM Tomography for the Detection of Ultra Small Gold Labels within Stained and Plastic-Embedded Sections of Tissue
}

\author{
Bram Koster, Ulrike Ziese, Willie Geerts, Theo van der Krift and Arie Verkleij \\ Molecular Cell Biology, Utrecht University, 3584 CH Utrecht, The Netherlands
}

Electron tomography is a three-dimensional (3D) imaging method with transmission electron microscopy (TEM) that provides high-resolution 3D images of structural arrangements [1,2]. With electron tomography a series of images is acquired of a sample that is tilted over a large angular range $\left( \pm 70^{\circ}\right)$ with small angular tilt increments. For the $3 \mathrm{D}$-reconstruction, the images of the tilt series are aligned relative to each other and the 3D-reconstruction is computed. Electron tomography is the only technique that can provide 3D information with nm-scale resolution of individual and unique samples.

A line of method development in our laboratory is directed to the optimization of electron tomography for applications on immunogold labeled, high-pressure frozen, freeze substituted, lowicryl-embedded and stained specimens. This type of electron microscope specimen preparation is highly suitable for questions for which 3D imaging of the general cellular architecture is required, or for questions for which invasive preparation steps for labeling with small gold is not a disadvantage. We envision that this will be a powerful approach for general questions within cell biology, structural biology as well as for and interactive genomics. Due to the required resources related to instrumentation, specimen preparation, 3D electron microscopy, and visualization, close collaboration between academic and industrial groups is crucial to bring this applicability of electron tomography up to a level that the scientific community can make use of the technique on a routine basis.

To further develop the methodology, all three fields of research - specimen preparation, data collection, data processing and analysis - will need to be optimized to being able to localize as a routine assay, proteins and lipids with high sensitivity and high reliability in tissue-material as well as in suspension. The required technological developments can be divided into three fields.

(1) Specimen preparation. A line of research is directed to the further development of robust and reproducible preparation methods that allow 3D immunogold labeling, e.g. by using small antibody fragments with high affinity (Llama antibody technology) and by tag specific (active or non-active) proteins and lipids. This approach includes coupling of ultra-small gold to llama-antibodies, and after labeling, to cryofix the specimens using high-pressure freezing and freeze substituted to preserve the ultrastructure optimally. Finally, thick sections of about $300 \mathrm{~nm}$ are made, which subsequently stained to visualize the global architecture such as membrane and cytoskeleton.

(2) Data collection. Optimization of automated high-resolution tomography data collection $[3,4,5]$. Combination of (Bright- or Dark-field) TEM or STEM imaging for detection of ultra small gold labels within stained and plastic-embedded sections of tissue [6].

(3) Data reconstruction and data analysis. Optimization of restoration approaches to improve the resolution of electron tomographic reconstructions by incorporating the image formation process (scattering within the specimen) and properties of the specimen (staining).

One of the key technological issues will be the capability to automatically detect ultra-small gold labels in tomograms of thick sections. Preliminary data suggests that HAADF-STEM tomography may well be a suitable mode for this purpose [6]. 


\section{References}

[1] J. Frank, Cur. Opin. Struct. Biol. 5(1995)194-201.

[2] W. Baumeister et al., Trends Cell Biol. 9 (1999) 81.

[3] K.D. Dierksen et al., Ultramicroscopy 40 (1992) 81.

[4] A.J. Koster et al., J. Structural Biol. 120 (1997) 276.

[5] Ziese et al. J Microsc. 205 (2002) 187.

[6] Ziese et al., J Struct Biol. 138:58-62.

[7] Research of A.J. Koster has been made possible by a fellowship of the Royal Netherlands Academy of Arts and Sciences (KNAW), the research of U. Ziese also by support from the KNAW and the research of W.J.C. Geerts by a collaborative grant of ALW Fysische Biologie program 2000/07115 with Prof.dr. Judith Klumperman (UMCU, Utrecht).

\section{STEM: 3D detection of $1 \mathrm{~nm}$ gold markers}

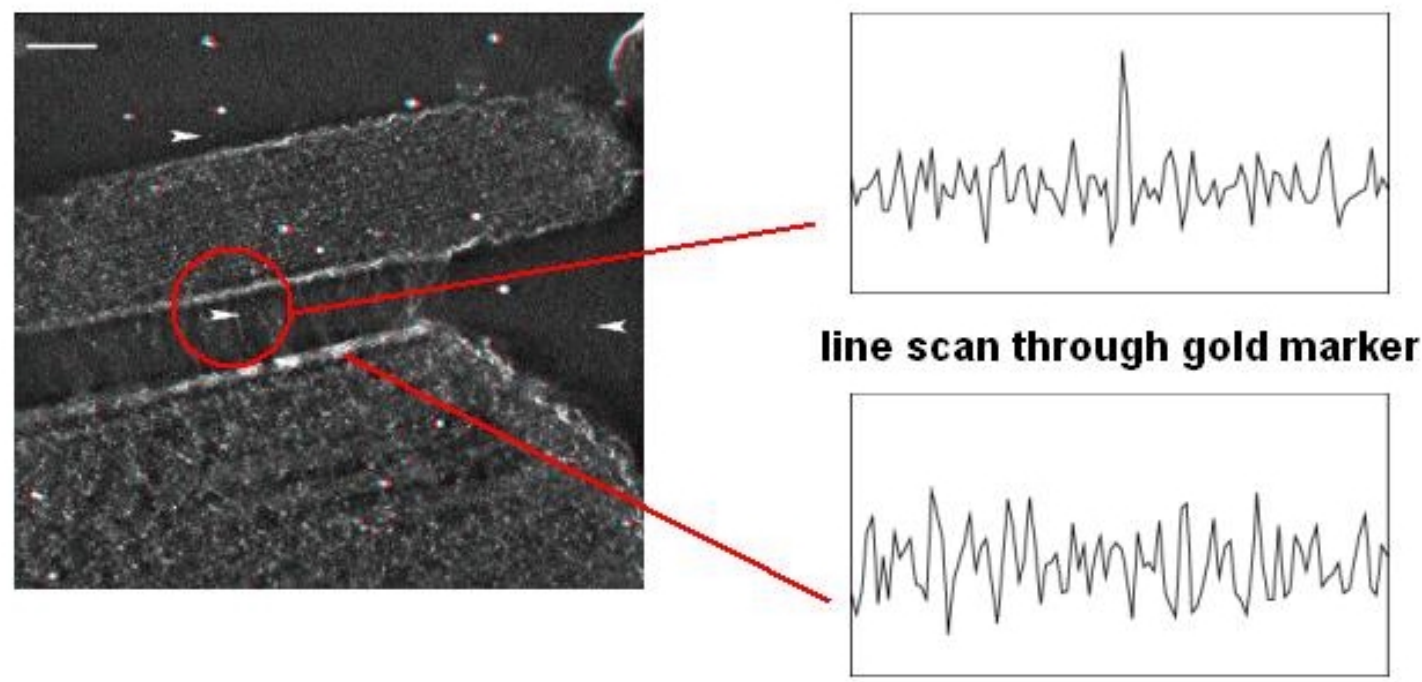

3D view of reconstruction

line scan through stained area

FIG. 1. We will discuss the current status of our approach to develop and implement the technical means to localize ultra-small gold labels within their cellular environment in our laboratory. We have implemented the data collection method at Utrecht University and TEM tiltseries are acquired with a $200 \mathrm{kV}$ Philips/FEI Tecnai $20 \mathrm{FEG}$ (S)TEM, equipped with a $2048 x 2048$ pixel slow-scan CCD camera (14 $\mu$ m per pixel, TemCam F214, TVIPS GmbH). The automation intelligence is implemented in JavaScript. 